Cerebrovasc Dis 2007;23:72-73

DOI: $10.1159 / 000097032$

\section{Clinical Trials and Dipyridamole Formulation Selection}

\section{Hartmut Derendorf}

College of Pharmacy, Pharmaceutics Department, University of Florida, Gainesville, Fla., USA

In a recent issue of Cerebrovascular Diseases, Jonas and Grieco [1] present a meta-analysis of several randomized secondary stroke prevention trials in patients with previous noncardioembolic stroke. The investigators conclude that 'antiplatelet therapy with aspirin plus dipyridamole is the best treatment yet studied for secondary stroke prevention after noncardioembolic stroke'. This conclusion agrees with the most recent results of the 5-year European/Australasian Stroke Prevention in Reversible Ischaemia Trial (ESPRIT) published late in May 2006, which found that aspirin and dipyridamole are more effective than aspirin alone in the prevention of new serious vascular events in patients after cerebral ischemia of arterial origin [2].

In contrast to the conclusions reached by the ESPRIT study group as well as recommendations from the Antithrombotic Trialists' Collaboration [2, 3], Jonas and Grieco [1] argue that the only remaining question from stroke prevention trials is 'which formulation of dipyridamole should be employed'. They propose using conventional dipyridamole at a total daily dose of $225 \mathrm{mg}$ (75 mg 3 times daily) combined with low-dose aspirin (once daily) for secondary stroke prevention after noncardioembolic stroke. Interestingly, the only trials that showed clinical benefit from aspirin plus dipyridamole combined treatment used extended-release formulations in doses of $200 \mathrm{mg}$ twice daily rather than conventional immediate-release tablets that are recommended by Jonas and Grieco.

A separate meta-analysis of 4 early studies in a total of 1,757 patients that examined effects of conventional dipyridamole found only a $3 \%$ added benefit from dipyridamole compared with aspirin that was not significant [4]. The European Stroke Prevention Trial 2 (ESPS2) was the first large trial to show a significant relative-risk reduction of all major vascular events for combined treatment with aspirin plus dipyridamole. Risk reduction was $22 \%$ (95\% confidence interval 9-33) compared with aspirin alone in the study with 3,299 patients [5]. The combination prevented twice as many strokes as aspirin alone. Similarly, the ESPRIT study in 2,739 patients randomized to aspirin (30-325 mg daily) or dipyridamole (200 mg twice daily) reported a significant difference in ischemic events that included death from all vascular causes, nonfatal stroke, nonfatal myocardial infarction or major bleeding complication. The incidence of outcome events was $13 \%$ with combination aspirin plus dipyridamole versus $16 \%$ with aspirin alone, i.e. an absolute risk reduction of 3\%, basically replicating ESPS2 findings [2]. ESPS2 used a composite extended-release buffered dipyridamole $(200 \mathrm{mg}$ ) formulation that combined immediate-release low-dose aspirin ( $25 \mathrm{mg}$ ), given twice daily. In the ESPRIT study, $83 \%$ of the patients randomized to dipyridamole received an extended-release formulation (200 mg twice daily) with a median $75 \mathrm{mg}$ daily dose of aspirin.

The conclusion of Jonas and Grieco [1] that conventional dipyridamole is as effective as extended-release dipyridamole formulations when combined with aspirin is not based on a demonstrated efficacy from clinical trial outcomes. Rather, justification for selecting a conventional dipyridamole tablet dosing regimen is based on 'a nonrandom sampling of retail cost in the United States' [1] while dipyridamole dose selection of a 3 times daily 75 mg tablet appears arbitrary. The authors imply that $225 \mathrm{mg} /$ day will decrease discontinuation due to headache, but provide no data showing dose or exposure relationships to this adverse event. Neither drug compliance nor bioavailability differences were considered. Compliance with a 3 times daily dosing regimen is certainly more difficult to maintain compared with twice daily dosing. Since bioavailability studies demonstrate that peak plasma drug concentrations are comparable following a conventional dipyridamole 100-mg tablet and a composite 200-mg extendedrelease tablet [6], it remains to be demonstrated whether a 75-mg immediate-release tablet will decrease the incidence of headache associated with dipyridamole discontinuation. Further, elevated gastric $\mathrm{pH}$, associated with commonly prescribed antacids and proton pump inhibitors, significantly impairs dipyridamole absorption from conventional formulations but has no effect on buffered extended-release formulations [7].

Optimized formulations that are designed to improve compliance and maintain drug exposure should not be dismissed as providing no benefit to patients. Results of aspirin plus dipyridamole large-scale trials demonstrated efficacy with optimized formulations and should be compared with studies that failed to show efficacy with conventional formulations, thereby guiding prescribing selection.

\section{References}

1 Jonas S, Grieco G: Dipyridamole plus aspirin: the best regimen for stroke prevention after noncardioembolic focal cerebral ischemia. Cerebrovasc Dis 2006;22:1-3.

2 The European/Australasian Stroke Prevention in Reversible Ischaemia Trial (ESPRIT) Study Group: Aspirin plus dipyridamole versus aspirin alone after cerebral ischaemia of arterial origin (ESPRIT): randomised controlled trial. Lancet 2006;367:1665-1673.

\section{KARGER}

Fax +41613061234 E-Mail karger@karger.ch www.karger.com
(C) 2007 S. Karger AG, Basel

$1015-9770 / 07 / 0231-0072 \$ 23.50 / 0$ 
3 Antithrombotic Trialists' Collaboration: Collaborative meta-analysis of randomised trials of antiplatelet therapy for prevention of death, myocardial infarction, and stroke in high risk patients. BMJ 2002;324: 71-86.

4 Algra A, van Gijn J, Koudstaal PJ: Secondary prevention after cerebral ischaemia of presumed arterial origin: is aspirin still the touchstone? J Neurol Neurosurg Psychiatry 1999;66:557-559.

5 European Stroke Prevention Study 2: efficacy and safety data. J Neurol Sci 1997;151(suppl):S1-S77.

6 Dresse A, Chevolet C, Delapierre D, Masset H, Weisenberger H, Bozler G, Heinzel G: Pharmacokinetics of oral dipyridamole (Persantine) and its effect on platelet adenosine uptake in man. Eur J Clin Pharmacol 1982;23:229-234

7 Derendorf H, Vander Maelen CP, Brickl RS, MacGregor TR, Eisert W: Dipyridamole bioavailability in subjects with reduced gastric acidity. J Clin Pharmacol 2005;45:845-850.

Hartmut Derendorf, PhD

Distinguished Professor and Chair, Department of Pharmaceutics

University of Florida, 100494, College of Pharmacy

Gainesville, FL 32610 (USA)

Tel. +1 352846 2726, Fax +1 3523923249

E-Mail Hartmut@cop.ufl.edu

Cerebrovasc Dis 2007;23:73-74

DOI: $10.1159 / 000097033$

\section{Reply to the Letter by Derendorf: Clinical Trials and Dipyridamole Formulation Selection}

G. Grieco, S. Jonas

Section of Quantitative Investigation, Department of Neurology, New York University School of Medicine, New York, N.Y., USA

Dr. Derendorf has offered a detailed critique of our editorial, and raised some interesting questions. After careful consideration, we must disagree with some of his observations and comments. In particular, we note:

(1) Dr. Derendorf quotes us as having stated that 'antiplatelet therapy with aspirin plus extended-release dipyridamole is the best treatment yet studied for secondary stroke prevention after noncardioembolic stroke'. However, we stated that 'antiplatelet therapy with dipyridamole plus aspirin is the best treatment yet studied for secondary stroke prevention after non-cardioembolic stroke' (emphases added).

(2) Dr. Derendorf states that 'Jonas and Grieco argue that the only remaining question from stroke prevention trials is "which formulation of dipyridamole should be employed"'. We would be foolhardy or naïve to believe that would be the only unanswered question; we are well aware of many other unresolved issues regarding stroke prevention. What we did say was that given that the best available evidence favors treatment with dipyridamole plus aspirin, what formulation of dipyridamole should be used?

(3) Dr. Derendorf argues that '... the only trials that showed clinical benefit from aspirin plus dipyridamole combined treat- ment used extended-release formulations in doses of $200 \mathrm{mg}$ twice daily rather than conventional immediate-release tablets that are recommended by Jonas and Grieco'. We are surprised by this conclusion. Our table shows the results of the AICLA and ESPS1 trials of conventional dipyridamole plus aspirin versus placebo. The relative risks of the individual trials and of the metaanalysis (0.581, 0.620 and 0.614 , respectively; all statistically significant) are remarkably similar to the relative risk (0.628) of extended-release dipyridamole plus aspirin versus placebo in the ESPS2 study.

(4) Dr. Derendorf states: 'A separate meta-analysis of 4 early studies in a total of 1,757 patients that examined effects of conventional dipyridamole found only a $3 \%$ added benefit from dipyridamole compared with aspirin that was not significant.' He does not explain that the endpoint was not stroke but a triple endpoint including stroke. He also does not indicate that one of the studies used a 150-mg total daily dipyridamole regimen, which is not a regimen for which we make a clinical claim, and not a regimen we included in our analyses. Furthermore, the reference for one of the studies is an unpublished 'Boehringer Ingelheim internal report'.

(5) We are baffled by the statement 'dipyridamole dose selection of a 3 times daily 75-mg tablet appears arbitrary'. To quote the ESPS2 Study Group in the introduction to the report of their study results: 'In 1987 the first ESPS study found that a combination of ASA (990 mg daily) and dipyridamole (225 mg daily) compared to placebo achieved the largest stroke risk reduction ever reported for stroke and TIA patients (38.1\%)' [1]. Our interest in a 225 -mg daily dipyridamole regimen is based on this important study sponsored, as was ESPS2, by Boehringer Ingelheim.

(6) On the question of headache, it would be nice to have the dose- and duration-related discontinuation data Dr. Derendorf asks for. Publicly available sources do not provide data from the ESPS1 trial on treatment discontinuations due to headache and other adverse events. For ESPS2 the discontinuation rates in the scientific literature and the product labeling do not agree. Thus, the ESPS2 group in their safety paper [2] reported that $8.0 \%$ of subjects on extended-release dipyridamole (with or without aspirin) dropped out because of headache, versus $2.1 \%$ of subjects on aspirin or placebo $(\mathrm{p}<0.001)$. The FDA-approved product labeling reports (in its table 3 ) discontinuation rates of $10 \%$ for both the Aggrenox ${ }^{\circledR}$ and extended-release dipyridamole groups, $3 \%$ for the aspirin group and $4 \%$ for the placebo group.

We stand by our comments on headaches. We made it clear that what we were saying was based on our clinical experience. This experience is consistent with the reported severalfold higher peak plasma concentrations attained with the extended-release formulation (see item 7a below) compared with a conventional formulation. It is also consistent with Boehringer Ingelheim's own conclusions on the association of headache severity and duration with plasma concentrations of dipyridamole [3].

(7) In support of the claimed superiority of extended-release versus conventional formulations, Dr. Derendorf cites the article he wrote [4], with 4 co-authors from Boehringer Ingelheim, comparing dipyridamole pharmacokinetics for Aggrenox and generic dipyridamole in 42- to 64-year-old normal subjects whose gastric $\mathrm{pH}$ was purposely raised to at least 4 by administration of a proton pump inhibitor. Ultimately, bioavailability differences do not matter when there is no difference in efficacy demonstrated in controlled trials. It is nonetheless interesting to note that while 